\title{
Cumulative Sum Control Charts for Simultaneously Monitoring Means and Variances of Multiple Quality Variables
}

\author{
Duk-Joon Chang1 and Sunyeong $\mathrm{Heo}^{2^{\dagger}}$
}

\begin{abstract}
Multivariate cumulative sum (CUSUM) control charts for simultaneously monitoring both means and variances under multivariate normal process are investigated. Performances of multivariate CUSUM schemes are evaluated for matched fixed sampling interval (FSI) and variable sampling interval (VSI) features in terms of average time to signal (ATS), average number of samples to signal (ANSS). Multivariate Shewhart charts are also considered to compare the properties of multivariate CUSUM charts. Numerical results show that presented CUSUM charts are more efficient than the corresponding Shewhart chart for small or moderate shifts and VSI feature with two sampling intervals is more efficient than FSI feature. When small changes in the production process have occurred, CUSUM chart with small reference values will be recommended in terms of the time to signal.
\end{abstract}

Key words : Likelihood Ratio Test (LRT) Statistic, Markov Chain Approach, Run Length, Time to Signal

\section{Introduction}

There are many situations in which the quality of a product or constancy of a process is determined by joint levels of several related quality variables. In this case, the quality of a product is usually characterized by joint levels of several related variables and a multivariate quality control procedure for simultaneously monitoring several related variables is needed.

The multivariate approach to quality control was first introduced by Hotelling ${ }^{[1]}$. Jackson ${ }^{[2]}$, and Ghare and Togersen $^{[3]}$ presented multivariate Shewhart control charts based on Hotelling's $T^{2}$ statistic. Alt ${ }^{[4]}$ described various types of Shewhart type $T^{2}$ charts and provided recommendations for implementation. Woodall and Ncube $^{[5]}$ considered a single multivariate CUSUM procedure for controlling the means of multivarite normal process. They described how a $p$ dimensional multivariate normal process can be monitored by using $p$

\footnotetext{
${ }^{1}$ Professor, Department of statistics, Changwon National University, Changwon 641-773, Korea

${ }^{2}$ Associate Professor, Department of statistics, Changwon National University, Changwon 641-773, Korea

†Corresponding author : syheo@changwon.ac.kr

(Received : September 18, 2012, Revised : December 15, 2012,

Accepted : December 21, 2012)
}

two-sided univariate CUSUM charts.

The ability of a control chart to detect process changes is determined by the length of time required for the chart to signal. In FSI chart, the expected time to signal is simply the product of the average run length (ARL) and the length of the fixed sampling interval.

Reynolds and Arnold ${ }^{[6]}$ investigated the theoretical aspects of one-sided VSI univariate Shewhart charts in detail. One disadvantage of VSI scheme is that frequent switching between different sampling intervals requires more cost and effort to administer the production process than corresponding FSI scheme. Amin and Letsinger ${ }^{[7]}$ showed that average number of switches to signal (ANSW) of the CUSUM procedure exists far fewer than that of the Shewhart procedure. According to the numerical performances of the considered charts, we also found that CUSUM procedure is more efficient than Shewhart procedure in terms of switching behaviors for both means and variances on multivariate procedure.

Most of the studies on multivariate control charts have been concentrated on monitoring mean vector of multivariate normal process. Before recent years, the use of multivariate quality control techniques was hampered by the lack of adequate computational resources. In this paper, we present multivariate control schemes to 
simultaneously monitor both means and variances of related quality variables, and evaluate and compare the performances of considered multivariate control charts.

\section{Multivariate Sample Statistics}

Assume that the process of interest has $p(p \geqq 2)$ related quality variables represented by the random vector $\underline{X}^{\prime}=\left(X_{1}, X_{2}, \cdots, X_{p}\right)$ and we obtain a sequence of random vectors $\underline{X}_{1}, \underline{X}_{2}, \cdots \quad$ where $\underline{X}^{\prime}{ }_{i}=\left(\underline{X}_{i 1}{ }^{\prime}, \underline{X}_{i 2}{ }^{\prime}, \cdots, \underline{X}_{i n}{ }^{\prime}\right)$ is a sample of observations at the sampling point $i \quad(i=1,2, \cdots)$ and $\underline{X}_{i j}{ }^{\prime}=\left(X_{i j 1}, X_{i j 2}, \cdots, X_{i j p}\right)$. Thus, $\underline{X}_{i}$ is an $n p \times 1$ vector. It will be assumed that the sequential observation vectors between and within samples are independent and identically distributed.

In this paper, we assume that process quality variables have a multivariate normal distribution. Hence, the distribution of $\underline{X}$ is indexed by a set of parameters $\underline{\theta}=(\underline{\mu}, \Sigma)$ where $\underline{\mu}$ is the mean vector and $\Sigma$ is the variance- covariance matrix of $\underline{X}$. Let $\underline{\theta}_{0}=\left(\underline{\mu}_{0}, \Sigma_{0}\right)$ be the known target values for $\underline{\theta}=(\underline{\mu}, \Sigma)$ of $p$ related quality variables and $\underline{\theta}_{0}$ is represented as

$$
\underline{\mu}_{0}=\left(\mu_{10}, \mu_{20}, \cdots, \mu_{p 0}\right)^{\prime}
$$

and

$$
\Sigma_{0}=\left[\begin{array}{cccc}
\sigma_{10}^{2} & \rho_{120} \sigma_{10} \sigma_{20} & \cdots & \rho_{1 p 0} \sigma_{10} \sigma_{p 0} \\
& \sigma_{20}^{2} & \cdots & \rho_{2 p 0} \sigma_{20} \sigma_{p 0} \\
& & \ddots & \vdots \\
& S y m & & \sigma_{p 0}^{2}
\end{array}\right],
$$

where the target covariance of variables $X_{r}$ and $X_{s}$ is $\sigma_{r s 0}=\rho_{r s 0} \sigma_{r 0} \sigma_{s 0}$ for $r, s=1,2, \cdots, p$. For simplicity in our computation, we assume that $\mu_{r 0}=0, \sigma_{r 0}^{2}=1.0$, $\rho_{r s 0}=0.4$ for $r, s=1,2, \cdots, p$.

To control both $\mu$ and $\sigma^{2}$ of single quality variable, Reynolds and Ghosh ${ }^{[8]}$ proposed an obvious method to use the statistic $W_{i}$ and the chart signals if

$$
W_{i}=\sum_{j=1}^{n}\left(X_{i j}-\mu_{0}\right)^{2} / \sigma_{0}^{2} \geqq \chi_{1-\alpha}^{2}(n),
$$

where $\chi_{1-\alpha}^{2}(p)$ is the $100(1-\alpha)$ th percentile of a chi-square distribution with $n$ degrees of freedom.
Extending the sample statistic $W_{i}$ to multivariate case, we obtain the sample statistic $D_{i}$ for multivariate control chart

$$
D_{i}=\sum_{j=1}^{n}\left(\underline{X}_{i j}-\underline{\mu}_{0}\right)^{\prime} \Sigma_{0}^{-1}\left(\underline{X}_{i j}-\underline{\mu}_{0}\right)
$$

If the process is in control, $D_{i}$ has a chi-squared distribution with $n p$ degrees of freedom. When the process has shifted to $\underline{\mu}$ from the target $\underline{\mu}_{0}, D_{i}$ has a non-central chi-squared distribution with $n p$ degrees of freedom with noncentrality parameter $\tau^{2}=n\left(\underline{\mu}-\underline{\mu_{0}}\right)^{\prime}$ $\Sigma_{0}^{-1}\left(\underline{\mu}-\underline{\mu_{0}}\right)$.

Another control chart for both $\mu$ and $\Sigma$ can be constructed by using both of the likelihood ratio test (LRT) statistic for testing $H_{0}: \mu=\mu_{0}$ vs $H_{1}: \underline{\mu} \neq \underline{\mu}_{0}$, where $\Sigma_{0}$ is known, and $H_{0}: \Sigma=\Sigma_{0}$ vs $H_{1}: \Sigma \neq \Sigma_{0}$ where $\underline{\mu}_{0}$ is known, respectively.

By simple calculation about likelihood ratio $\lambda$ at the $i$ th sample $(i=1,2, \cdots)$ for testing $H_{0}: \underline{\mu}=\underline{\mu}_{0}$ vs $H_{1}: \underline{\mu} \neq \underline{\mu}_{0}$ where $\Sigma_{0}$ is known, we obtain multivariate sample statistic $T M_{i}$ for monitoring means. The LRT statistic $T M_{i}$ is given by

$$
T M_{i}=n\left(\underline{\bar{X}}_{i}-\underline{\mu}_{0}\right)^{\prime} \Sigma_{0}^{-1}\left(\underline{\bar{X}}_{i}-\underline{\mu}_{0}\right) .
$$

Similarly, LRT statistic $T V_{i}$ for variances is

$$
\begin{aligned}
T V_{i}=\operatorname{tr}\left(A_{i} \Sigma_{0}^{-1}\right) & -n \ln \left|A_{i}\right|+n \ln \left|\Sigma_{0}\right| . \\
& +n p \ln n-n p
\end{aligned}
$$

where $V_{i}=\sum_{j=1}^{n}\left(\underline{X}_{i j}-\bar{X}_{i}\right)^{\prime} \Sigma_{0}^{-1}\left(\underline{X}_{i j}-\underline{X_{i}}\right)$.

Thus, LRT statistics $T M_{i}$ and $T V_{i}$ can be used as sample statistics for monitoring $\underline{\mu}$ and $\Sigma$, respectively. Since the statistic $T M_{i}$ has a chi-square distribution with $p$ degrees of freedom, the percentage point of $T M_{i}$ can be obtained from a chi-square distribution. But it is difficult to obtain the exact distribution of $T V_{i}$. Thus, the percentage points of $T V_{i}$ can be obtained by simulations.

A multivariate Shewhart chart based on $D_{i}$ signals whenever $D_{i} \geqq \chi_{1-\alpha}^{2}(n p)$. And a multivariate Shewhart control chart based on $\left(T M_{i}, T V_{i}\right)$ uses the separate chart for $\underline{\mu}$ and $\Sigma$, and signals if one of the two charts signals when 


$$
T M_{i} \geqq \chi_{1-\alpha_{\underline{\mu}}}^{2}(p) \text { or } T V_{i} \geq h_{T V},
$$

where the parameter $h_{T V}$ can be obtained by simulation.

\section{FSI Schemes}

Shewhart chart, although simple to understand and apply, uses only the information in the current sample and is thus relatively inefficient in detecting small changes on process parameters.

To overcome this difficulty, Page ${ }^{[9]}$ investigated the properties of warning lines used within the control limits of a Shewhart chart in order to incorporate the information from the past few samples. He suggested that if $r$ out of the last $N$ samples fell between the warning lines and control limits, an action should be taken. He showed that the Shewhart chart with warning lines is more efficient in detecting small shifts than the standard Shewhart chart.

The CUSUM control chart was first proposed by Page $^{[9]}$. Page ${ }^{[10,11]}$ and Barnard ${ }^{[12]}$ developed the CUSUM procedure as a sequential likelihood ratio test for testing the hypothesis that the process mean is equal to the target value against the alternative that it is not. Hawkins ${ }^{[13]}$ proposed a CUSUM chart for monitoring a standard deviation, primarily for the case in which only individual observation at each occasion is available.

The CUSUM control chart is a good alternative to the Shewhart control chart when small shifts are important. The CUSUM chart directly incorporates all of the information in the sequence of sample values by plotting the cumulative sums of the deviation of the sample values from the target value.

One traditional practice in using a control chart is to take samples from the process at FSI and properties of control charts have been developed when the sampling interval between samples is fixed. In FSI control chart, the length of the sampling interval between sampling times $t_{i}$ and $t_{i-1}$, is constant for all $i(i=1,2, \cdots)$.

A multivariate FSI CUSUM based on the statistic $D_{i}$ at the $i$ th sample is

$$
Y_{D, i}=\max \left\{Y_{D, i-1}, 0\right\}+\left(D_{i}-k_{D}\right),
$$

where $Y_{D, 0}=\omega \cdot I_{(\omega \geqq 0)}, k_{D} \geqq 0$. This chart signals whenever $Y_{D, i} \geqq h_{D}$.

When the parameters are on-target or $\underline{\mu}$ has shifted, the properties of the multivariate CUSUM chart based on $D_{i}$ can be evaluated by the Markov chain approach or integral equation method. When the process shifts in $\Sigma$, or $\underline{\mu}$ and $\Sigma$, the properties of the chart can be evaluated by simulation.

For simultaneous use of LRT statistics $\left(T M_{i}, T V_{i}\right)$ given in (2.2) and (2.3), the multivariate FSI CUSUM control scheme for $\underline{\mu}$ at the $i$ th sample is

$$
Y_{T M, i}=\max \left\{Y_{T M, i-1}, 0\right\}+\left(T M_{i}-k_{T M}\right)
$$

and for $\Sigma$ at the $i$ th sample is

$$
Y_{T V, i}=\max \left\{Y_{T V, i-1}, 0\right\}+\left(T V_{i}-k_{T V}\right),
$$

where $\quad k_{T V} \geqq 0, \quad Y_{T M, 0}=\omega_{T M} \cdot I_{\left(\omega_{T M} \geqq 0\right)} \quad$ and $Y_{T V, 0}=\omega_{T V} \cdot I_{\left(\omega_{T V} \geqq 0\right)}$. This chart signals whenever $Y_{T M, i} \geqq h_{T M}$ or $Y_{T V, i} \geqq h_{T V}$.

Since it is difficult to obtain the exact joint distribution of (3.2) and (3.3), the performances of this scheme can be evaluated by simulation when the parameters of the process are on-target or changed.

\section{VSI Schemes}

Recent years, application of VSI control procedure has become quite frequent and several papers have been published. The basic idea of a VSI control chart is that the time sampling interval should be short if there is an indication of a process change and should be long if there is no indication of a process change. In VSI chart, it is necessary to keep track of both ATS and ANSS. Following the definition of Reynolds et al. ${ }^{[14]}$, the number of samples to signal (NSS) is taken from the start of the process to the time when the chart signals and ANSS is the expected value of the number of samples to signal (NSS). Also, they defined that the time to signal (TS) is taken from the start of the process to the time when the chart signals and ATS is the expected value of the TS. Therefore, NSS in VSI scheme has the same definition as run length (RL) in FSI scheme.

For the VSI CUSUM chart based on $D_{i}$ in (2.1), suppose that the sampling interval ; 
Table 1. Performances of multivariate charts based on $D_{i}$ when $\mu_{1}$ has changed

\begin{tabular}{ccccccccc}
\hline & \multicolumn{2}{c}{ Shewhart } & \multicolumn{2}{c}{ CUSUM $\left(k_{D}=9.5\right)$} & \multicolumn{2}{c}{ CUSUM $\left(k_{D}=10.0\right)$} & \multicolumn{2}{c}{ CUSUM $\left(k_{D}=10.5\right)$} \\
\cline { 2 - 8 } & ANSS & ATS & ANSS & ATS & ANSS & ATS & ANSS & ATS \\
\hline in control & 200.00 & 200.00 & 200.00 & 200.00 & 200.00 & 200.00 & 200.00 & 200.00 \\
\hline$M_{1}$ & 159.11 & 152.87 & 124.14 & 110.47 & 129.60 & 117.84 & 134.62 & 124.28 \\
$M_{2}$ & 88.67 & 75.54 & 46.61 & 31.86 & 48.80 & 34.32 & 52.65 & 38.58 \\
$M_{3}$ & 41.70 & 29.17 & 20.16 & 11.92 & 19.23 & 10.91 & 19.77 & 11.32 \\
$M_{4}$ & 19.03 & 10.38 & 11.08 & 6.50 & 9.84 & 5.43 & 9.46 & 5.11 \\
$M_{5}$ & 9.14 & 4.00 & 7.09 & 4.34 & 6.08 & 3.60 & 5.62 & 3.31 \\
$M_{6}$ & 4.83 & 1.97 & 4.99 & 3.26 & 4.21 & 2.78 & 3.81 & 2.59 \\
$M_{7}$ & 2.86 & 1.34 & 3.75 & 2.67 & 3.14 & 2.37 & 2.82 & 2.26 \\
$M_{8}$ & 1.92 & 1.13 & 2.96 & 2.33 & 2.48 & 2.15 & 2.21 & 2.09 \\
$M_{9}$ & 1.44 & 1.05 & 2.43 & 2.14 & 2.03 & 2.04 & 1.81 & 2.00 \\
$M_{10}$ & 1.20 & 1.02 & 2.05 & 2.04 & 1.72 & 1.98 & 1.53 & 1.96 \\
$M_{11}$ & 1.08 & 1.01 & 1.77 & 1.99 & 1.47 & 1.95 & 1.32 & 1.93 \\
$M_{12}$ & 1.03 & 1.00 & 1.55 & 1.96 & 1.28 & 1.93 & 1.17 & 1.92 \\
\hline
\end{tabular}

Table 2. Performances of multivariate charts based on $\left(T M_{i}, T V_{i}\right)$ when $\mu_{1}$ has changed

\begin{tabular}{ccccccccc}
\hline & \multicolumn{2}{c}{ Shewhart chart } & \multicolumn{3}{c}{ CUSUM } & \multicolumn{3}{c}{ CUSUM } \\
& \multicolumn{3}{c}{$\left.k_{T M}=3.5, k_{T V}=11.5\right)$} & \multicolumn{4}{c}{ CUSUM } \\
\cline { 2 - 8 } & ANSS & ATS & ANSS & ATS & ANSS & ATS & ANSS & ATS \\
\hline in control & 200.02 & 200.00 & 199.99 & 200.00 & 199.96 & 199.98 & 199.96 & 199.97 \\
\hline$M_{1}$ & 152.55 & 146.06 & 116.09 & 103.81 & 127.13 & 115.63 & 134.13 & 123.84 \\
$M_{2}$ & 73.60 & 62.01 & 34.05 & 24.35 & 38.11 & 26.94 & 43.56 & 31.90 \\
$M_{3}$ & 30.49 & 20.69 & 13.20 & 8.56 & 12.76 & 7.43 & 13.68 & 7.75 \\
$M_{4}$ & 12.46 & 6.62 & 7.15 & 4.35 & 6.34 & 3.44 & 6.28 & 3.20 \\
$M_{5}$ & 5.80 & 2.64 & 4.60 & 2.74 & 3.93 & 2.13 & 3.72 & 1.92 \\
$M_{6}$ & 3.17 & 1.50 & 3.29 & 1.91 & 2.77 & 1.54 & 2.57 & 1.43 \\
$M_{7}$ & 2.02 & 1.17 & 2.52 & 1.47 & 2.12 & 1.26 & 1.95 & 1.19 \\
$M_{8}$ & 1.47 & 1.06 & 2.02 & 1.24 & 1.70 & 1.12 & 1.56 & 1.09 \\
$M_{9}$ & 1.20 & 1.02 & 1.68 & 1.11 & 1.42 & 1.06 & 1.32 & 1.04 \\
$M_{10}$ & 1.08 & 1.01 & 1.43 & 1.06 & 1.23 & 1.03 & 1.16 & 1.02 \\
$M_{11}$ & 1.03 & 1.00 & 1.25 & 1.03 & 1.11 & 1.01 & 1.06 & 1.01 \\
$M_{12}$ & 1.01 & 1.00 & 1.12 & 1.01 & 1.04 & 1.00 & 1.02 & 1.00 \\
\hline
\end{tabular}

Table 3. Performances of multivariate charts based on $D_{i}$ when $\sigma_{1}^{2}$ has changed

\begin{tabular}{ccccccccc}
\hline & \multicolumn{2}{c}{ Shewhart } & \multicolumn{2}{c}{ CUSUM $\left(k_{D}=9.5\right)$} & \multicolumn{2}{c}{ CUSUM $\left(k_{D}=10.0\right)$} & \multicolumn{2}{c}{ CUSUM $\left(k_{D}=10.5\right)$} \\
\cline { 2 - 8 } & ANSS & ATS & ANSS & ATS & ANSS & ATS & ANSS & ATS \\
\hline in control & 200.00 & 200.00 & 200.00 & 200.00 & 200.00 & 200.00 & 200.00 & 200.00 \\
\hline$V_{1}$ & 109.71 & 99.54 & 68.97 & 51.40 & 72.20 & 56.28 & 76.44 & 61.74 \\
$V_{2}$ & 58.55 & 48.04 & 33.84 & 21.13 & 34.11 & 21.49 & 35.78 & 23.64 \\
$V_{3}$ & 33.29 & 24.85 & 21.09 & 11.94 & 20.09 & 11.06 & 20.26 & 11.47 \\
$V_{4}$ & 20.32 & 13.95 & 14.83 & 8.20 & 13.50 & 7.10 & 13.19 & 6.98 \\
$V_{5}$ & 13.46 & 8.62 & 11.29 & 6.26 & 10.01 & 5.17 & 9.52 & 4.86 \\
$V_{6}$ & 9.40 & 5.71 & 9.04 & 4.92 & 7.91 & 4.01 & 7.36 & 3.73 \\
$V_{7}$ & 6.90 & 4.08 & 7.47 & 4.10 & 6.45 & 3.33 & 5.97 & 3.05 \\
$V_{8}$ & 5.43 & 3.16 & 6.35 & 3.53 & 5.46 & 2.88 & 5.02 & 2.62 \\
$V_{9}$ & 4.37 & 2.54 & 5.52 & 3.12 & 4.71 & 2.55 & 4.30 & 2.30 \\
$V_{10}$ & 3.66 & 2.15 & 4.86 & 2.78 & 4.13 & 2.28 & 3.76 & 2.06 \\
$V_{11}$ & 3.15 & 1.89 & 4.34 & 2.53 & 3.69 & 2.08 & 3.37 & 1.89 \\
$V_{12}$ & 2.76 & 1.70 & 3.91 & 2.30 & 3.35 & 1.92 & 3.04 & 1.76 \\
\hline
\end{tabular}


Table 4. Performances of multivariate charts based on $\left(T M_{i}, T V_{i}\right)$ when $\sigma_{1}^{2}$ has changed

\begin{tabular}{|c|c|c|c|c|c|c|c|c|}
\hline & \multicolumn{2}{|c|}{ Shewhart chart } & \multicolumn{2}{|c|}{$\begin{array}{c}\text { CUSUM } \\
\left(k_{T M}=3.5, k_{T V}=11.5\right)\end{array}$} & \multicolumn{2}{|c|}{$\begin{array}{c}\text { CUSUM } \\
\left(k_{T H}=4.0, k_{T V}=12.0\right)\end{array}$} & \multicolumn{2}{|c|}{$\begin{array}{c}\text { CUSUM } \\
\left(k_{T M}=4.5, k_{T V}=12.5\right)\end{array}$} \\
\hline & ANSS & ATS & ANSS & ATS & ANSS & ATS & ANSS & ATS \\
\hline in control & 200.02 & 200.00 & 199.99 & 200.00 & 199.96 & 199.98 & 199.96 & 199.97 \\
\hline$V_{1}$ & 149.62 & 143.97 & 122.06 & 109.41 & 128.95 & 117.81 & 133.68 & 123.83 \\
\hline$V_{2}$ & 102.88 & 94.53 & 71.14 & 57.26 & 76.39 & 62.84 & 81.85 & 69.01 \\
\hline$V_{3}$ & 68.04 & 59.58 & 45.16 & 33.14 & 47.35 & 35.03 & 50.36 & 38.37 \\
\hline$V_{4}^{3}$ & 46.44 & 38.56 & 31.06 & 22.01 & 31.12 & 21.27 & 32.75 & 22.72 \\
\hline$V_{5}$ & 32.42 & 25.49 & 22.87 & 15.79 & 22.30 & 14.46 & 23.00 & 14.82 \\
\hline$V_{6}$ & 23.35 & 17.45 & 17.90 & 12.20 & 16.88 & 10.59 & 17.05 & 10.40 \\
\hline$V_{7}$ & 17.53 & 12.48 & 14.48 & 9.88 & 13.29 & 8.18 & 13.15 & 7.77 \\
\hline$V_{8}$ & 13.36 & 9.12 & 12.13 & 8.30 & 10.85 & 6.63 & 10.52 & 6.13 \\
\hline$\stackrel{\circ}{V}_{9}$ & 10.70 & 7.01 & 10.35 & 7.04 & 9.10 & 5.61 & 8.73 & 5.07 \\
\hline$V_{10}$ & 8.70 & 5.51 & 8.98 & 6.15 & 7.85 & 4.88 & 7.40 & 4.33 \\
\hline$V_{11}$ & 7.25 & 4.49 & 7.92 & 5.52 & 6.85 & 4.33 & 6.45 & 3.79 \\
\hline$V_{12}^{11}$ & 6.17 & 3.75 & 7.08 & 4.98 & 6.12 & 3.83 & 5.69 & 3.35 \\
\hline
\end{tabular}

Table 5. Performances of multivariate charts based on $D_{i}$ when $\mu_{1}$ and $\sigma_{1}^{2}$ have changed

\begin{tabular}{ccccccccc}
\hline & \multicolumn{3}{c}{ Shewhart } & \multicolumn{2}{c}{ CUSUM $\left(k_{D}=9.5\right)$} & \multicolumn{2}{c}{ CUSUM $\left(k_{D}=10.0\right)$} & \multicolumn{2}{c}{ CUSUM $\left(k_{D}=10.5\right)$} \\
\cline { 2 - 8 } & ANSS & ATS & ANSS & ATS & ANSS & ATS & ANSS & ATS \\
\hline in control & 200.00 & 200.00 & 200.00 & 200.00 & 200.00 & 200.00 & 200.00 & 200.00 \\
$\left(M_{1}, V_{1}\right)$ & 87.71 & 76.60 & 51.37 & 35.48 & 53.71 & 38.28 & 57.53 & 42.87 \\
$\left(M_{2}, V_{2}\right)$ & 30.94 & 22.36 & 19.17 & 10.75 & 17.99 & 9.71 & 18.05 & 9.88 \\
$\left(M_{3}, V_{3}\right)$ & 12.17 & 7.33 & 10.18 & 5.45 & 8.92 & 4.40 & 8.48 & 4.10 \\
$\left(M_{4}, V_{4}\right)$ & 5.97 & 3.24 & 6.46 & 3.43 & 5.55 & 2.77 & 5.11 & 2.52 \\
$\left(M_{5}, V_{5}\right)$ & 3.49 & 1.93 & 4.59 & 2.49 & 3.90 & 2.03 & 3.52 & 1.85 \\
$\left(M_{6}, V_{6}\right)$ & 2.41 & 1.45 & 3.50 & 1.96 & 2.98 & 1.65 & 2.70 & 1.50 \\
$\left(M_{7}, V_{7}\right)$ & 1.86 & 1.24 & 2.81 & 1.62 & 2.40 & 1.41 & 2.19 & 1.33 \\
$\left(M_{8}, V_{8}\right)$ & 1.54 & 1.14 & 2.35 & 1.43 & 2.02 & 1.28 & 1.84 & 1.21 \\
$\left(M_{9}, V_{9}\right)$ & 1.36 & 1.08 & 2.02 & 1.30 & 1.74 & 1.19 & 1.61 & 1.14 \\
$\left(M_{10}, V_{10}\right)$ & 1.25 & 1.05 & 1.77 & 1.21 & 1.55 & 1.13 & 1.44 & 1.10 \\
$\left(M_{11}, V_{11}\right)$ & 1.17 & 1.04 & 1.59 & 1.15 & 1.42 & 1.09 & 1.33 & 1.07 \\
$\left(M_{12}, M_{12}\right)$ & 1.12 & 1.03 & 1.45 & 1.11 & 1.32 & 1.07 & 1.24 & 1.05 \\
\hline
\end{tabular}

Table 6. Performances of multivariate charts based on $\left(T M_{i}, T V_{i}\right)$ when $\mu_{1}$ and $\sigma_{1}^{2}$ have changed

\begin{tabular}{ccccccccc}
\hline & \multicolumn{2}{c}{ Shewhart chart } & \multicolumn{3}{c}{ CUSUM } & \multicolumn{3}{c}{ CUSUM } \\
& ANSS & ATS & ANSS & ATS & ANSS & \multicolumn{3}{c}{ CUSUM } \\
\cline { 2 - 8 } & ANS & ANS & ANSS & ATS \\
\hline in control & 200.02 & 200.00 & 199.99 & 200.00 & 199.96 & 199.98 & 199.96 & 199.97 \\
$\left(M_{1}, V_{1}\right)$ & 108.75 & 100.38 & 72.72 & 58.99 & 79.62 & 66.52 & 85.28 & 73.16 \\
$\left(M_{2}, V_{2}\right)$ & 36.35 & 29.01 & 21.44 & 15.11 & 21.76 & 14.55 & 23.47 & 15.80 \\
$\left(M_{3}, V_{3}\right)$ & 13.57 & 9.20 & 9.95 & 6.72 & 9.05 & 5.66 & 9.08 & 5.46 \\
$\left(M_{4}, V_{4}\right)$ & 6.42 & 3.93 & 5.97 & 4.07 & 5.23 & 3.31 & 5.04 & 3.06 \\
$\left(M_{5}, V_{5}\right)$ & 3.79 & 2.26 & 4.12 & 2.83 & 3.55 & 2.32 & 3.34 & 2.12 \\
$\left(M_{6}, V_{6}\right)$ & 2.59 & 1.63 & 3.11 & 2.18 & 2.67 & 1.81 & 2.50 & 1.67 \\
$\left(M_{7}, V_{7}\right)$ & 1.99 & 1.34 & 2.48 & 1.79 & 2.16 & 1.53 & 2.02 & 1.43 \\
$\left(M_{8}, V_{8}\right)$ & 1.65 & 1.20 & 2.08 & 1.55 & 1.83 & 1.37 & 1.72 & 1.29 \\
$\left(M_{9}, V_{9}\right)$ & 1.45 & 1.13 & 1.80 & 1.39 & 1.60 & 1.26 & 1.52 & 1.20 \\
$\left(M_{10}, V_{10}\right)$ & 1.31 & 1.08 & 1.60 & 1.28 & 1.45 & 1.18 & 1.39 & 1.14 \\
$\left(M_{11}, V_{11}\right)$ & 1.22 & 1.05 & 1.46 & 1.21 & 1.33 & 1.13 & 1.28 & 1.10 \\
$\left(M_{12}, M_{12}\right)$ & 1.16 & 1.04 & 1.35 & 1.15 & 1.25 & 1.09 & 1.21 & 1.07 \\
\hline
\end{tabular}


$d_{1}$ is used when $Y_{D, i} \in\left(g_{D}, h_{D}\right]$,

$d_{2}$ is used when $Y_{D, i} \in\left(-k_{D,} g_{D}\right]$,

where $-k_{D}<g_{D} \leqq h_{D}$ and $d_{1}<d_{2}$. And for the VSI scheme based on $\left(T M_{i}, T V_{i}\right)$ in $(2.2)$ and (2.3), the sampling interval ;

$$
\begin{aligned}
& d_{1} \text { is used when } Y_{T M, i} \in\left(g_{T M}, h_{T M}\right] \\
& \quad \text { or } Y_{T V, i} \in\left(g_{T V}, h_{T V}\right], \\
& d_{2} \text { is used when } Y_{T M, i} \in\left(-k_{T M} g_{T M}\right] \\
& \quad \text { or } Y_{T V, i} \in\left(-k_{T V} g_{T V}\right],
\end{aligned}
$$

where the parameters $-k_{T M}<g_{T M} \leqq h_{T M}$ and $-k_{T V}<g_{T V} \leqq h_{T V}$. The parameters $h_{D}, g_{D}, h_{T M}$ and $g_{T M}$ can be obtained by Markov chain approach, and the parameters $h_{T V}$ and $g_{T V}$ can be obtained by simulation.

\section{Numerical Results and Conclusion}

In order to evaluate the performances and compare the matched FSI and VSI multivariate CUSUM and Shewhart charts, some kinds of standards for comparison are necessary.

For convenience, we let that the sampling interval of unit time $d$ be 1.0 in FSI charts and two sampling intervals used as $d_{1}=0.1$ and $d_{2}=1.9$ for the VSI charts. And we let the sampling interval used before the first sample, say $d_{0}$ be 1.0 for all considered VSI schemes. In our computation, ANSS and ATS of the considered charts when the process is in controlwere fixed to be 200 and the sample size $n$ for each variable is 3 for $p=3$. The types of shifts in the process parameters when the process is out of control for comparison can be stated as follows :

1) Shifts in means : $M_{i}$, one mean $\mu_{1}$ is shifted to $\tau^{2}=(0.5 \cdot i)^{2}, i=1,2, \cdots, 12$.

2) Shifts in variances : $V_{i}$, one variance $\sigma_{1}^{2}$ is increased to $(1.0+0.1 \cdot i)^{2}, i=1,2, \cdots, 12$.

3) Shifts in means and variances : $\left(M_{i}, V_{i}\right)$, $i=1,2, \cdots, 12$.

After the reference values of the multivariate CUSUM schemes have been determined, the parameters $h$ and $g$ values based on $D_{i}$ and $T M_{i}$ were calculated by Markov chain method with transition states $r=100$. And the parameters $h$ and $g$ values based on LRT statistic $T V_{i}$, and ANSS and ATS for the proposed types of shifts for Shewhart and CUSUM charts were obtained by simulation with 10,000 iterations. The numerical results were stated in Table 1 through Table 6 .

According to the numerical performances in Table 1 through Table 6, we can notice the followings. When some shifts of the peocess means have occurred, the multivariate chart based on statistics $\left(T M_{i}, T V_{i}\right)$ is more efficient than the multivariate chart based on $D_{i}$ but the difference is small. When some shifts of the peocess variances have occurred, the multivariate chart based on statistic $D_{i}$ is more efficient than the chart based on statistics $\left(T M_{i}, T V_{i}\right)$, and the difference in this case is larger than the difference in the shift of means.

When small or moderate changes for both means and variances in the process have occurred, multivariate CUSUM chart based on $D_{i}$ is more efficient than multivariate Shewhart chart in our computation. Numerical results for various reference values show that large reference values are efficient for large shifts and smaller reference values are efficient for small shifts in multivariate CUSUM charts. And for the proposed multivariate control charts and all kinds of shifts, VSI scheme is more efficient than FSI scheme.

\section{References}

[1] H. Hotelling, "Multivariate quality control", Techniques of statistical analysis, McGraw -Hill, New York, pp. 111-184, 1947.

[2] J. S. Jackson, "Quality control methods for several related variables", Technometrics, Vol. 1, pp. 359377, 1959.

[3] P. H. Ghare and P. E. Torgersen, "The multicharacteristic control chart”, J. Ind. Eng., Vol. 19, pp. 269-272, 1968.

[4] F. B. Alt, "Multivariate quality control", in The encyclopedia of statistical sciences, eds. S. Kotz and Johnson, John Wiley, New York, 1982.

[5] W. H. Woodall and M. M. Ncube, "Multivariate CUSUM quality control procedure", Technometrics, Vol. 27, pp. 285-292, 1985.

[6] M. R. Jr. Reynolds and J. C. Arnold, "Optimal 
one-sided Shewhart control charts with variable sampling intervals between samples", Sequential Analysis, Vol. 8, pp. 51-77, 1989.

[7] R. W. Amin and W. C. Letsinger, "Improved switching rules in control procedures using variable sampling intervals", Commun. Stat.-Simul. C., Vol. 20, pp. 205-230, 1991.

[8] M. R. Jr. Reynolds and B. K. Ghosh, "Designing control charts for means and variances", ASQC Quality Congress Transactions, San Francisco, pp. 400-407, 1981.

[9] E, S. Page, "Control charts with warning lines", Biometrika, Vol. 42, pp. 243-259, 1955.
[10] E. S. Page, "Continuous inspection schemes", Biometrika, Vol. 41, pp. 100-114, 1954.

[11] E, S. Page, "Cumulative sum charts", Techometrics, Vol. 3, pp. 1-9, 1961.

[12] G. A. Barnard, "Control charts and stochastic process”, J. R. Stat. Soc. B, Vol. 21, pp. 239-257, 1959.

[13] D. M. Hawkins, (1981), "A Cusum for a Scale Parameter", J. Qual. Technol., Vol. 13, pp. 228-231, 1981.

[14] M. R. Jr. Reynolds, R. W. Amin, J. C. Arnold, and J. A Nachlas, " $\bar{X}$-charts with variable sampling intervals", Technometrics, Vol. 30, pp. 181-192, 1988. 